\title{
Chart review across EU5 in MM post-ASCT patients
}

\author{
John Ashcroft*,1, Davneet Judge ${ }^{2}$, Sujith Dhanasiri ${ }^{3}$, Gavin Taylor-Stokes ${ }^{2} \&$ Chloe \\ Middleton ${ }^{2}$ \\ ${ }^{1}$ Department of Haematology, Mid-Yorkshire NHS Trust, Wakefield, WF1 4DG2, UK \\ ${ }^{2}$ Adelphi Real World, Bollington, Cheshire, SK10 5JB, UK \\ ${ }^{3}$ Celgene International, Boudry, CH-2017, Switzerland \\ *Author for correspondence: John.ashcroft@midyorks.nhs.uk
}

\begin{abstract}
Aim: To understand the current treatment patterns, clinical outcomes and healthcare resource utilizationassociated costs for multiple myeloma patients, post autologous stem cell transplant (ASCT) across Europe. Patients \& methods: Medical records were used to abstract data for 337 multiple myeloma patients who had received ASCT. Results: Following ASCT, 7\% received maintenance therapy prior to progression. Lenalidomide was the most frequently prescribed maintenance, second- and third-line therapy. Monthly resource use was considerably lower in patients who received maintenance therapy (€638.14 vs $€ 1001.74)$. Median time to progression was longer for patients who had received maintenance therapy. Conclusion: The study highlights the diversity in current treatment patterns post-ASCT. Results suggest patients who receive maintenance therapy have a prolonged remission period, and as a result their associated healthcare resource utilization is spread across the treatment pathway.
\end{abstract}

First draft submitted: 11 April 2018; Accepted for publication: 18 June 2018; Published online: 11 July 2018

Keywords: healthcare resource utilization • lenalidomide $\bullet$ maintenance treatment $\bullet$ multiple myeloma $\bullet$ real world - stem cell transplantation • treatment patterns

The recent introduction of novel molecular agents has increased the therapeutic armory in multiple myeloma (MM). In addition, new roles for established agents are also being developed, as evidenced by the recent approval of lenalidomide as maintenance therapy following high-dose chemotherapy and autologous stem cell transplant (ASCT). ASCT following induction treatment remains the global gold standard treatment for eligible MM patients [1,2]. Maintenance therapy has shown substantial improvements in survival outcomes (e.g., response rates, progression-free survival [PFS], time to progression [TTP] and overall survival) versus conventional chemotherapies and placebo for MM patients $[3,4,5,6,7,8]$. It is suggested that maintenance treatments control the proliferation of residual malignant cells after transplantation and have demonstrated their benefit by controlling the disease for longer periods of time $[8,9]$. The effectiveness of these novel therapies used either as single agents or in combination has produced a paradigm shift in the way MM is treated [6]. However, uncertainties remain about optimum treatment strategies and with an ever-increasing choice of agents, the best sequence of available treatment options has not been fully established [10]. Current clinical practice guidelines within Europe, USA and Canada recommend induction followed by high-dose therapy with stem cell support and lenalidomide maintenance treatment $[3,11]$. The introduction of newer treatment options also impacts on the resource use and economic burden of this disease.

Lenalidomide (Revlimid ${ }^{\circledR}$ ), a thalidomide analog, is the first treatment to be indicated for maintenance treatment for patients with MM in the post-ASCT setting. It received approval, in February 2017, based on two large Phase III randomized clinical trials showing approximately 15-month (the Cancer and Leukemia Group B [CALGB] trial) and 18-month (the Intergroupe Francophone du Myelome (IFM) 2005-02 trial) PFS advantages in patients who received maintenance treatment with lenalidomide versus placebo (i.e., no treatment post-ASCT) $[9,12,13,14,15]$. In these studies, lenalidomide demonstrated a good tolerability profile, an advantage over some other agents, such as the parent drug thalidomide, which is limited by neurotoxicity during long-term use. While clinical trials provide

Future Medicine 
key data regarding the efficacy and safety of new therapies, real-world studies are also required to establish the benefit of new therapies in routine clinical practice.

In addition to the efficacy and safety associated with a treatment, it is also important to consider the costs to healthcare service providers and patients. Market access decisions require further detailed economic evaluations providing information on treatment patterns, and costs associated with resource use. The substantial economic burden of MM is well documented $[16,17,18]$. The disease- and treatment-related costs associated with managing MM are considerable; drug treatments and hospitalizations have been shown to be the largest cost components [16]. Although ASCT is the standard of therapy to achieve remission in MM, there is a considerable personal and economic burden associated with the procedure itself and what it means to the patient and the healthcare system. For example, initial therapy often includes three to six cycles of induction therapy prior to transplant; the collection of stem cells procedure is costly, complex, and time consuming, and involves the coordination of various departments and healthcare professionals (HCPs); and a second transplant may also be necessary in patients who do not achieve complete remission $[19,20]$. In one study, ASCT accounted for more than $80 \%$ of the nonpharmacological treatment costs [16]. Furthermore, considerable amounts of time and resources are necessary for the management of patients and the financial and time constraints of the patients themselves and their caregivers can be substantial [21].

Few studies have assessed the costs associated with novel agent-based treatment regimens in MM. Recently, in an analysis using data from the Connect MM registry [33], there were nonsignificant trends toward a shorter mean duration of hospitalization (5.0 vs 10.5 days in Year 1; 7.0 vs 9.0 days in Year 2) and lower rates of overall hospitalizations and use of certain medications (e.g., neuropathic pain treatments) in patients receiving lenalidomide maintenance therapy compared with patients who received no maintenance treatment [22]. Therefore, in order to support decision making with regard to MM treatment, more research is needed into the real-world treatment pathway for MM patients; with an emphasis on whether the use of maintenance therapy has PFS advantages and the potential impact the use of such maintenance therapy has on the healthcare resource utilization (HCRU) costs associated with MM.

The objectives of this study are to describe and understand the current treatment practice patterns across the patient pathway as well as clinical outcomes (TTP and time to death) and HCRU-associated costs for patients with MM who have undergone ASCT in five European countries, as there is limited literature regarding these aspects of therapy.

\section{Methods}

Study design

A multicenter, retrospective observational chart review was performed for patients with MM who had received an ASCT as part of first-line treatment (Figure 1). The study was conducted in five European countries (France, Germany, Italy, Spain and UK) and involved physicians responsible for the management of patients with MM. Recruited physicians completed a medical chart review for 6-10 consecutive consulting patients who had undergone an ASCT as part of their first-line treatment on or after the index date of 1 January 2014. Data were extracted retrospectively from until January/March 2017 from individual patient charts and were recorded on electronic case report forms (eCRFs).

\section{Site/investigator selection}

A master list of investigators involved in the treatment and management of patients with MM was created in each country. The list of investigators consists of physicians who voluntarily wish to participate in observational research; all physicians within the list are systematically vetted against authoritative sources in each country, such as the General Medical Council (GMC) (UK) or equivalent. An eligible sample of physicians is selected based on study eligibility and willingness to participate. Although the results do not generalize to the entire MM population, the study recruited a geographically distributed sample of treating physicians who identified eligible patients, thus providing a broad overview of current MM practice.

Physicians expressing an interest in participating in the study were screened to ensure they met the following eligibility criteria: having one of the following specialisms - hematologist, hematology-oncology or internist; having been qualified for at least 5 years and no more than 38 years; spending at least $50 \%$ of their time managing patients in clinical practice, overall; and being responsible for and actively involved in the treatment management of at least three MM patients in a typical working week. Participating physicians selected patients meeting the following 


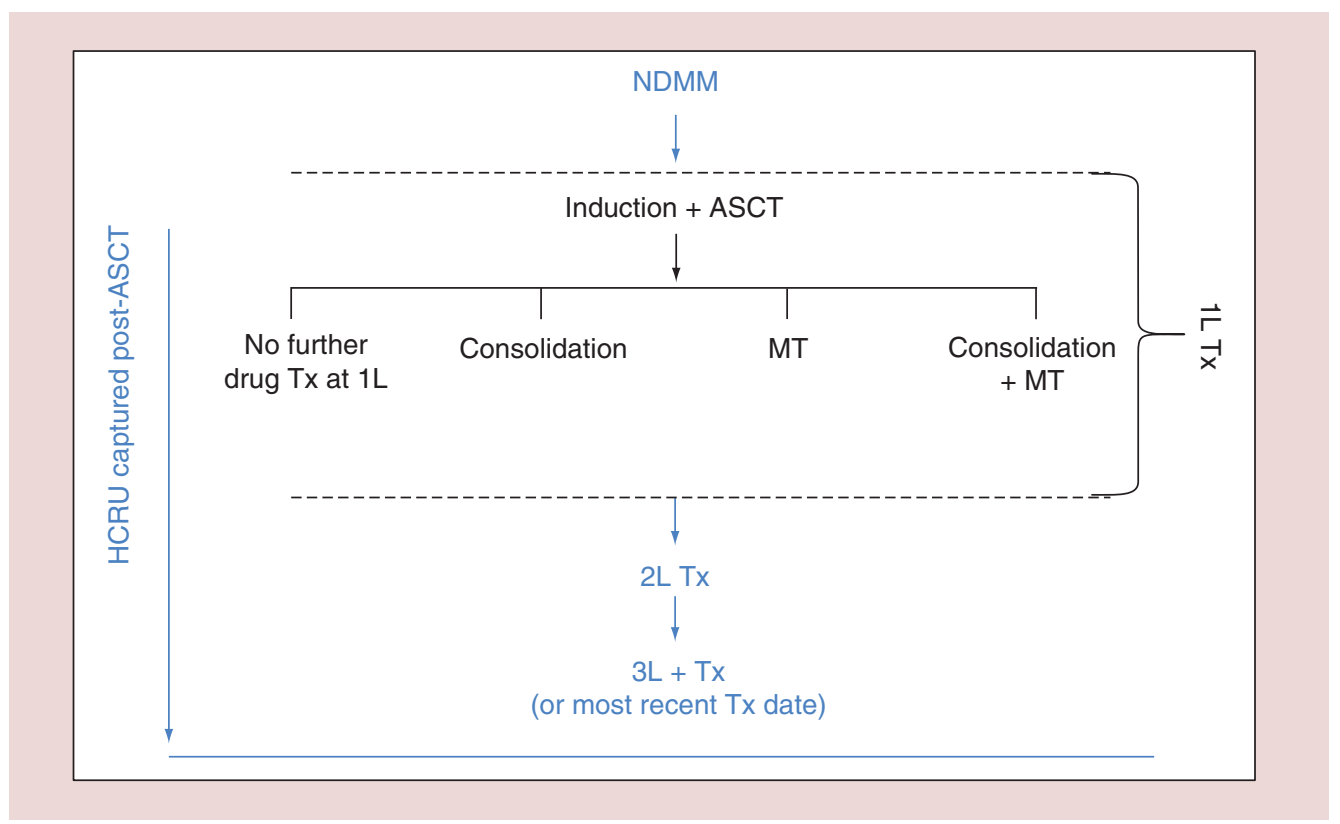

Figure 1. Treatment pathway flow for patient sample, detailing healthcare resource utilization capture. 1L: First-line; ASCT: Autologous stem cell transplant; HCRU: Healthcare resource utilization; MT: Maintenance therapy; NDMM: Newly diagnosed multiple myeloma; Tx: Treatment.

inclusion criteria: aged $\geq 18$ years, having a confirmed diagnosis of $\mathrm{MM}$ and having received induction drug therapy and an ASCT as part of their first-line treatment.

\section{Outcome measures}

The following data were collected for each patient: demographics, time to death (if applicable), details of first-line post-transplant treatment including types of therapy received (i.e., consolidation therapy - defined as treatments prescribed for a short period of time to improve ASCT response quality - and maintenance therapy - defined as a longer duration of treatment given to prolong the duration of remission/response effects); the following variables were collected across both first and second line of therapy: duration of therapy, TTP and HCRU (including total hospitalizations, additional supportive treatments, HCP visits and monitoring tests).

\section{HCRU costing analysis}

HCRU data were collected for each line of treatment line. First-line HCRU included resources used from completion of ASCT until initiation of second-line therapy; second-line HCRU included resources used from start of second line treatment to most recent date (third line, date of death and survey end). A unit analysis was used to calculate HCRU for each observation; over the course of each patient's treatment pathway, from completion of ASCT as part of first-line therapy to the most recent treatment date. Units were determined per month for all patients, and results were described as HCRU units per patient. Monthly resource use was calculated for the duration of patient follow-up.

Unit costs were applied to the relevant HCRU data to estimate the associated costs. Tariffs from the UK health service were initially applied (an exchange rate of $£ 1$ to $€ 1.1365$ was used; correct as of 1 July 2017). Costs for hospitalizations, supportive treatments, HCP visits and monitoring tests were obtained from the National Health Service Schedule of Reference Costs (2015-2016) and supportive drug costs were obtained from the Monthly Index of Medical Specialities 2017. The costs were applied to data collected from the healthcare system perspective thus allowing for a broad overview to assess the impact of therapy on HCRU across treatment lines following first-line ASCT. Equivalent unit costs for France, Germany, Italy and Spain were also obtained and used to determine total costs for these countries for comparison. The costs of active drug therapies were not included within the HCRU costing analysis. Costs for France, Germany, Italy and Spain were also obtained for comparison (see Appendix 1). 


\section{Costing assumptions}

When a hospitalization was recorded, emergency room and intensive care unit admission was captured and a one-off cost for the respective admission was applied (admission costs should be considered a minimum value as costs could be higher, depending on the number of days spent in the intensive care unit, for example). When an unspecified test was recorded, the lowest cost for a monitoring test was applied. Costs for prednisolone/prednisone were only available for the combination with either bendamustine or thalidomide; when patients were prescribed prednisolone/prednisone without either listed combination drug, the lowest cost was used. When body surface area (derived using the De Bois formula) or patient weight was required to calculate drug dosage, the average height and weight for male and female patients receiving an ASCT was taken from the Adelphi Disease Specific Programme Multiple Myeloma 2015-2015 data (Male: $174.6 \mathrm{~cm}$ and $77.5 \mathrm{~kg}$; Female: $163.3 \mathrm{~cm}$ and $70.1 \mathrm{~kg}$ was assumed for each gender). Cycle lengths were not accurately abstracted from eCRF data; therefore, standard drug cycles were assumed, and when a standard cycle length was not available, a 28-day cycle was assumed.

Data management and statistical analyses were conducted using Version 15.0 of the Stata software package (StataCorp LLC, TX, USA) [25].

\section{Statistical methods}

For all study objectives, standard descriptive statistics were used to describe the outcomes. Numerical variables were described in terms of mean, median and standard deviation (SD); categorical variables were described as frequency and percentage of patients falling into each category. A Kaplan-Meier approach was used to determine median TTP for first- and second-line therapy. For first-line therapy, TTP was considered to be the time from undergoing ASCT until the start of second-line therapy. For second-line therapy, the TTP was calculated as the period from the start of second-line therapy to the start of third-line therapy. Time to death was analyzed using the mean number of months between date of diagnosis and date of death.

\section{Ethical conduct of the study}

The study was conducted in accordance with the International Society for Pharmacoepidemiology and Guidelines for Good Pharmacoepidemiology Practices. The protocol was approved by the Freiburger Ethic-Komission International centralized ethics committee.

\section{Results}

Study population: physician \& patient characteristics

A total of 337 eCRFs were completed across the five countries by 51 physicians (number of physicians by country: ten in France, 11 in Germany, ten in Italy, ten in Spain and ten in the UK). The majority of physicians included were hematologists (69\%) and they saw an average of $20 \mathrm{MM}$ patients per week. A summary of the main patient demographic characteristics is presented by those who had received maintenance therapy and those who did not in Table 1. Overall, there were slightly more male than female patients in the study population ( $57 \mathrm{vs} 43 \%)$ and the mean (SD) age at diagnosis was $58.7( \pm 7.7)$ years. A total of $53 \%$ of patients had stage III MM at diagnosis, and $34 \%$ had stage II disease at diagnosis; the mean (SD) duration from diagnosis to ASCT was $9.8( \pm 13.2)$ months. Most patients (93\%) were alive at the time of data collection; the small proportion of deceased patients (7\%) had a mean $(\mathrm{SD})$ time to death of 22.4 ( \pm 12.6) months. Patients' demographics were similar across the cohort from each country.

Mean follow-up time for patients was 18.7 months from their initial ASCT; 21.2 and 18.5 months for maintenance and nonmaintenance therapy patients, respectively.

\section{Treatment patterns \& lines of therapy}

Following the first ASCT and as part of first-line therapy (i.e., until receiving second-line therapy), $71 \%$ of patients $(\mathrm{n}=240)$ did not receive any further drug therapy; $21 \%(\mathrm{n}=72)$ received consolidation therapy only, and 5\% $(n=16)$ received maintenance therapy only as part of their first-line treatment. In total, $3 \%$ of patients received both consolidation and maintenance therapy; therefore, overall $24 \%$ of patients $(n=81)$ received consolidation therapy and $7 \%(\mathrm{n}=25)$ received maintenance therapy. Higher usage of maintenance therapy was seen in France, Germany and Spain (6-9\%) compared with UK and Italy.

During the observation period (from initial post-ASCT treatment to the most recent treatment date) overall, $61 \%$ of patients received only one line of treatment, $27 \%$ received a total of two lines and $12 \%$ three or more 
Table 1. Demographic characteristics.

\begin{tabular}{|c|c|c|c|}
\hline Variable & All & Maintenance patients & Nonmaintenance patients \\
\hline $\mathrm{n}$ & 337 & 25 & 312 \\
\hline \multicolumn{4}{|c|}{ Age at diagnosis, years } \\
\hline Mean (SD) & $58.7(7.7)$ & $58.9(8.0)$ & $58.6(7.7)$ \\
\hline \multicolumn{4}{|l|}{ Sex, n (\%) } \\
\hline Male & $192(57)$ & $15(60)$ & $177(57)$ \\
\hline Female & $145(43)$ & $10(40)$ & $135(43)$ \\
\hline \multicolumn{4}{|c|}{ MM stage at diagnosis, $\mathrm{n}(\%)$} \\
\hline Smouldering & $9(3)$ & $2(8)$ & $7(2)$ \\
\hline Stage I & $32(9)$ & $1(4)$ & $31(10)$ \\
\hline Stage II & $113(34)$ & $8(32)$ & $105(34)$ \\
\hline Stage III & $180(53)$ & $13(52)$ & $167(54)$ \\
\hline Unknown & $3(1)$ & $1(4)$ & $2(1)$ \\
\hline \multicolumn{4}{|c|}{ Time from diagnosis to ASCT, months } \\
\hline Mean (SD) & $9.8(13.2)$ & $8.4(6.9)$ & $10.0(13.6)$ \\
\hline \multicolumn{4}{|c|}{ Living status, $\mathrm{n}(\%)$} \\
\hline Alive & $315(93)$ & $25(100)$ & $290(93)$ \\
\hline Dead & $22(7)$ & - & $22(7)$ \\
\hline \multicolumn{4}{|c|}{ Country, n (\%) } \\
\hline France & $73(22)$ & $7(28)$ & $66(21)$ \\
\hline Italy & $60(18)$ & $1(4)$ & $59(19)$ \\
\hline Germany & $74(22)$ & $9(36)$ & $65(21)$ \\
\hline Spain & $69(20)$ & $6(24)$ & $63(20)$ \\
\hline UK & $61(18)$ & $2(8)$ & $59(19)$ \\
\hline
\end{tabular}

\begin{tabular}{|c|c|c|c|}
\hline $\begin{array}{l}\text { Maximum number of lines received to } \\
\text { date, } n(\%)\end{array}$ & All $(n=337)$ & Maintenance patients $(n=25)$ & Nonmaintenance patients $(n=312)$ \\
\hline 1 line & $207(61)$ & $20(80)$ & $187(60)$ \\
\hline 2 lines & $91(27)$ & $5(20)$ & $86(28)$ \\
\hline 3 lines & $32(9)$ & 0 & $32(10)$ \\
\hline 4 lines & $6(2)$ & 0 & $6(2)$ \\
\hline
\end{tabular}

lines; 7\% received a second ASCT. Patients who received maintenance therapy (maintenance patients) were, on average, more likely to receive a lower number of treatment lines compared with patients who did not receive any maintenance therapy at first-line (nonmaintenance patients; 1.2 vs 1.6 lines, respectively). In total, $40 \%$ of nonmaintenance patients went on to receive two or more lines of drug therapy; whereas only $20 \%$ of maintenance patients progressed to second-line therapy, and none progressed to third line (Table 2).

\section{Overview of therapies \\ Treatment regimens}

Most patients who received maintenance therapy at first line were prescribed lenalidomide monotherapy (88\%); $8 \%$ received bortezomib and $4 \%$ received thalidomide. A thalidomide plus bortezomib combination was the most frequently prescribed consolidation treatment in nonmaintenance patients (68\%). Lenalidomide was the most frequently prescribed therapy across both second-line and third-line for nonmaintenance patients.

Lenalidomide-based regimens were the most frequently prescribed treatments for both maintenance and nonmaintenance patients ( 40 and 59\%, respectively) at second line, and for nonmaintenance patients at third line $(26 \%)$. None of the patients who received maintenance therapy at first line progressed to third line. 


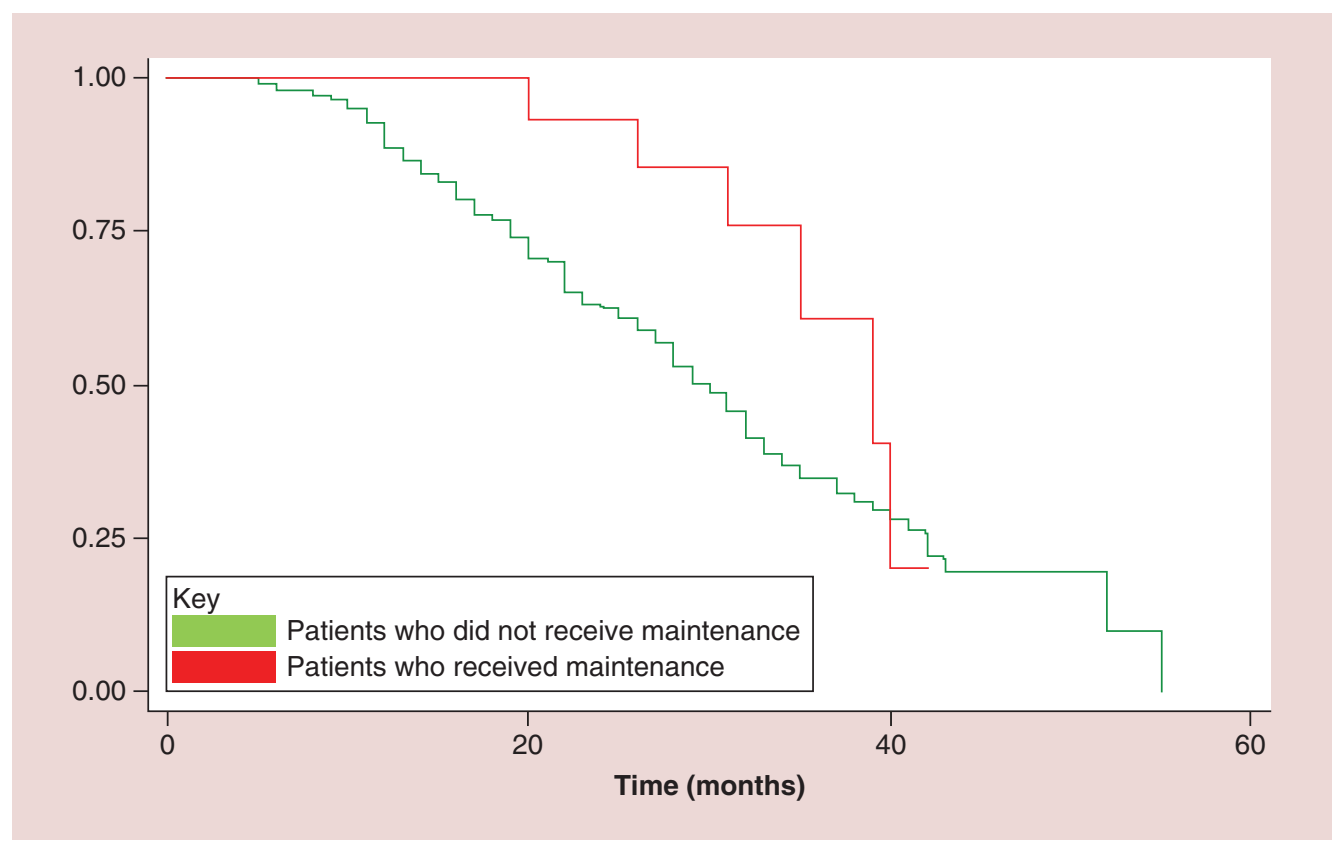

Figure 2. Kaplan-Meier chart showing the time to progression from first-line treatment.

\section{Duration of response}

TTP was longer for maintenance patients compared with nonmaintenance patients: 39.0 months for maintenance patients (95\% confidence interval: $31-$ not reached [months]; $\mathrm{n}=25)$ and 30.0 months for nonmaintenance patients (95\% confidence interval: $27-32$ [months]; $\mathrm{n}=312$ ) (Figure 2).

For nonmaintenance patients, the estimated median TTP from the start of second-line treatment was 13 months $(\mathrm{n}=104)$. Only five maintenance patients were reported to be receiving second-line therapy, as a result of the low base and no progression from second line, an estimated median TTP could not be calculated for these patients. In total, $13 \%$ of these patients at third line $(n=5)$ took a mean duration of 3.6 months to disease progression (an estimated median TTP could not be calculated owing to a low sample size).

\section{Supportive treatments}

Over the course of first-line treatment following ASCT, the mean number of different supportive therapies given was 3.48 overall: 6.36 for maintenance patients and 3.25 for nonmaintenance patients. The most frequently prescribed supportive treatments were antibiotics (given to $60 \%$ of patients overall: $72 \%$ of maintenance patients and 59\% of nonmaintenance patients), followed by bisphosphonates (54\% overall: $68 \%$ maintenance and $53 \%$ nonmaintenance) and analgesics (50\% overall: $72 \%$ maintenance and $45 \%$ nonmaintenance). All patients who were given maintenance therapy received at least one type of supportive therapy; whereas $15 \%$ of nonmaintenance patients did not receive any supportive treatments during first-line therapy.

The most popularly prescribed supportive treatments and the most frequently conducted monitoring tests were the same across all three treatment lines. Similar to the first-line results described above, the most frequently prescribed supportive treatments over the course of second and third lines of treatment were antibiotics ( $48 \%$ at second line; $62 \%$ at third line) and analgesics ( $42 \%$ at second line; $31 \%$ at third line). In total, $16 \%$ of all patients at second line and $18 \%$ of all patients at third line did not require any supportive treatments.

\section{HCRU-associated costs}

Of the 337 patients, 91 (14 France, 22 Germany, 17 Italy, 26 Spain and 12 UK) had the complete resource use data across their treatment pathway and had a follow-up time of 15 months. These 91 patients had a mean age of 60 (SD 7.7), and 55\% were male. Units were calculated per month for all patients (patients with missing data were excluded from unit resource calculations) for hospitalizations, HCP visits and monitoring tests. Across the observed resources, nonmaintenance patients had a higher number of HCRU units per month versus maintenance patients 
Non-MT $\square \mathrm{MT}$

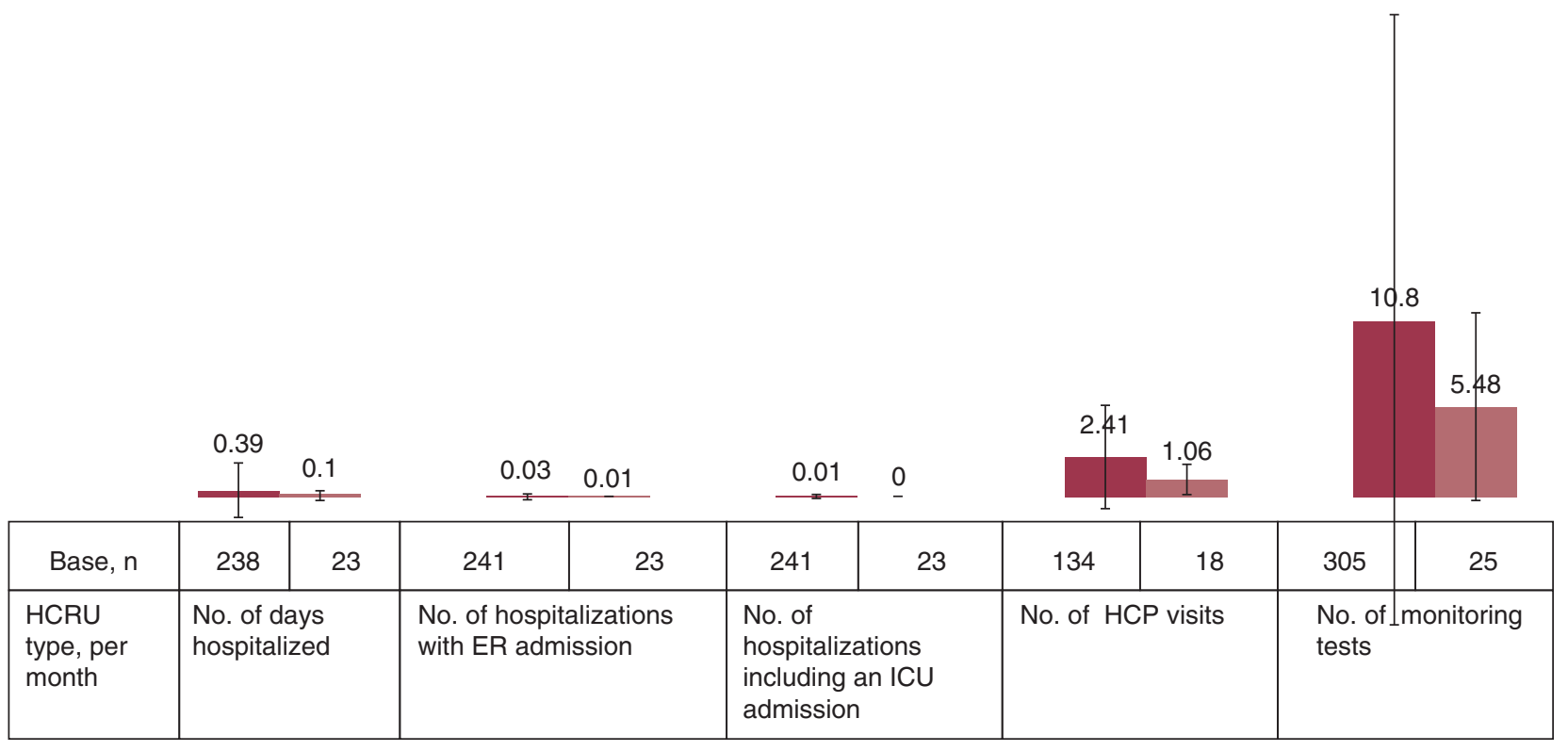

Figure 3. Healthcare resource utilization unit analysis: units calculated per month over the course of the patient's treatment pathway, from post autologous stem cell transplant at first-line onwards.

ER: Emergency room; HCP: Healthcare professional; HCRU: Healthcare resource utilization; ICU: Intensive care unit.

Table 3. Healthcare resource utilization-associated costs by maintenance and nonmaintenance patients.

Cost per month across treatment pathway, $€$

All $(n=337)$

Maintenance patients $(n=25) \quad$ Nonmaintenance patients $(n=312)$

\begin{tabular}{|c|c|c|c|c|c|c|}
\hline & & & & \\
\hline & $\mathrm{n}$ & Mean (SD) $€$ & n & Mean (SD) $€$ & $\mathrm{n}$ & Mean (SD) $€$ \\
\hline Total HCRU & 91 & $945.80(1060.88)$ & 14 & $638.14(547.80)$ & 77 & $1001.74(1122.88)$ \\
\hline Hospitalizations $^{\dagger}$ & 261 & $226.97(1001.56)$ & 23 & $59.78(131.00)$ & 238 & $243.12(1046.86)$ \\
\hline Supportive drugs ${ }^{\ddagger}$ & 328 & $264.81(580.95)$ & 25 & $442.52(626.51)$ & 303 & $250.15(575.69)$ \\
\hline Supportive treatments $\S$ & 330 & $24.68(113.96)$ & 25 & $55.12(193.21)$ & 305 & $22.18(105.00)$ \\
\hline HCP visits & 152 & $460.99(670.66)$ & 18 & $181.11(192.23)$ & 134 & $498.58(702.79)$ \\
\hline Monitoring tests & 203 & $187.22(328.00)$ & 20 & $84.20(72.81)$ & 183 & $198.48(342.87)$ \\
\hline
\end{tabular}

† Including ER and ICU admissions.

¥Including bisphosphonates (IV/oral), steroids, low molecular weight heparin, aspirin and denosumab.

$\S$ Including blood transfusions and dialysis.

ER: Emergency room; HCP: Healthcare professional; HCRU: Healthcare resource utilization; ICU: Intensive care unit; IV: Intravenous; SD: Standard deviation.

(Figure 3). The total cost of HCRU following first-line ASCT was €945.80 per patient per month (excluding the cost of active drug therapies). Patients receiving maintenance therapy ( $\mathrm{n}=14)$ had an associated HCRU cost of $€ 638.14$ per month compared with $€ 1001.74$ per patient per month for nonmaintenance patients $(\mathrm{n}=77)$.

The type of resource use recorded differed between the two groups (Table 3). Compared with nonmaintenance patients, maintenance patients incurred a greater expenditure on supportive drugs (€442.52 vs €250.15 per patient, respectively) and other supportive treatments ( $€ 55.12$ vs $€ 22.18$, respectively). However, expenditure on hospitalization, HCP visits and monitoring tests was higher for nonmaintenance patients (hospitalizations: $€ 243.12$ vs $€ 59.78$; HCP visits: $€ 498.58$ vs $€ 181.11$; monitoring tests: $€ 198.48$ vs $€ 84.20$ per patient, respectively).

Applying tariffs for the other four countries revealed a similar pattern of greater total costs being incurred in nonmaintenance versus maintenance patients (Table 4). The lowest total cost was observed when using the tariffs for Italy and the highest cost was observed when applying the tariffs from the UK. 
Table 4. Total healthcare resource utilization-associated costs by maintenance and nonmaintenance patients and by country.

\begin{tabular}{|c|c|c|c|c|c|c|}
\hline \multirow[t]{2}{*}{ Total cost per month across treatment pathway, $€$} & \multicolumn{2}{|c|}{ All $(n=337)$} & \multicolumn{2}{|c|}{ Maintenance patients $(n=25)$} & \multicolumn{2}{|c|}{ Nonmaintenance patients $(n=312)$} \\
\hline & $\mathbf{n}$ & Mean (SD) $€$ & $\mathbf{n}$ & Mean $(S D) €$ & m & Mean $(S D) €$ \\
\hline UK tariff analysis & 91 & $945.80(1060.88)$ & 14 & $638.14(547.80)$ & 77 & $1001.74(1122.88)$ \\
\hline France tariff analysis & 91 & $446.51(810.25)$ & 14 & $260.86(235.50)$ & 77 & $480.26(872.04)$ \\
\hline Germany tariff analysis & 91 & $562.58(1005.59)$ & 14 & $402.86(412.07)$ & 77 & $591.62(1078.37)$ \\
\hline Italy tariff analysis & 91 & $407.96(453.77)$ & 14 & $238.29(222.78)$ & 77 & $438.81(478.63)$ \\
\hline Spain tariff analysis & 91 & $620.07(943.31)$ & 14 & $301.00(219.56)$ & 77 & $678.08(1011.61)$ \\
\hline
\end{tabular}

HCRU: Healthcare resource utilization; SD: Standard deviation.

\section{Discussion}

The introduction of novel agents such as lenalidomide as maintenance therapy after transplant represented a major advance in the management of MM; it is therefore timely to review how clinicians are employing the available therapies and the benefits for their patients $[6,7,8]$. Real-world data describing the management of MM patients are limited and therefore this study reports treatment practice patterns, patient clinical outcomes and HCRU for a representative cohort of patients receiving treatment for MM in a real-world setting across five European countries. Additionally, recently conducted clinical studies (CALGB and IFM) have proven the longer PFS value of maintenance treatment, namely lenalidomide; however, the cost of this extended remission period on resource use (i.e., supportive therapies, hospital visits and monitoring) also requires consideration. An appreciation of costs and resource use are pivotal to ensuring appropriate use of available resources to enable patients to have access to new therapies and are able to benefit from developments in the management of this disorder.

This study provides valuable insights into current management of MM patients eligible to receive ASCT in Europe, including clinical outcomes and resource use, thus contributing to the published literature which aims to clarify some of these issues. In particular, this study reveals that maintenance therapy following ASCT is now entering routine clinical practice and provides clinical meaningful benefits, in agreement with the results from clinical trials. The study also reveals that maintenance therapy can delay progression to further lines of therapy and reduce resource use - outcomes that are likely to facilitate the management of MM.

The patients included in this study are considered to be representative of patients undergoing ASCT in the five European countries, as minimal screening criteria were implemented for both patients and physicians.

Additionally, the patient samples observed in this real-world study are reflective of the patient demographics data reported in Raab et al. 2016, another large European chart review of MM patients completed by treating physicians, thus the findings from this study can be extrapolated to routine clinical practice across Europe [10]. Owing to the small sample of maintenance patients in the current study, statistical analysis could not be carried out to compare patients who did and did not receive first-line maintenance therapy post-ASCT. As a result, descriptive statistical analysis was completed and any comparative results should be interpreted with caution.

There were a number of notable findings. Following the initial ASCT, 71\% of the total study samples did not receive any drug therapy; $7 \%$ of patients $(n=25)$ received maintenance therapy and in most cases $(88 \%)$ this was lenalidomide. Only $20 \%$ of maintenance therapy patients progressed to second-line therapy, and none progressed to third line in the observation period. Median TTP was 9 months longer in patients who received maintenance therapy post-ASCT compared with those who did not (median TTP of 39 vs 30 months, respectively). The TTP data for patients not receiving maintenance therapy (30 months) are comparable to recently published pooled PFS data from large randomized controlled trials (25 months) [26]. A retrospective chart review, conducted by Yang et al. 2017, identified a PFS of 41.7 months for MM patients who had received lenalidomide maintenance therapy post-ASCT [27]. According to McCarthy et al. 2012, although a cure is currently not possible in most patients, maintenance of a prolonged progression-free interval is an important goal in MM management [14]. Additionally, TTP decreases with increasing lines of therapy, which supports the rationale for delaying progression to later lines of therapy with maintenance treatment.

The results of this study also highlight that there is a continued HCRU impact post-ASCT, even if patients are not receiving any active ongoing treatment (i.e., maintenance therapy). The study results presented suggested that across the treatment pathway, total monthly HCRU-associated costs were lower in maintenance compared with nonmaintenance patients. HCP visits account for a significant proportion of HCRU costs, particularly for 
nonmaintenance patients and hospitalizations account for $20 \%$ of costs for nonmaintenance patients, whereas this cost is $<10 \%$ of total costs for maintenance patients. The lower hospitalization resource use for maintenance patients highlights the implication of reduced pressure on hospital beds and HCP in-patient services going forward. This same pattern was observed across markets when each country's respective costs were applied. The HCRU costing data observed within this study need to be taken into consideration with the cost of any active drug therapies that the patient may be receiving. However, as resource use was captured across the entire follow-up period, we are unable to derive whether an intense period of resource use occurred at a specific time point.

Overall, treatment patterns of the patient cohort in this study are generally comparable to those reported previously; for example, in the Raab et al. 2016 chart review nearly half of the 7635 included patients (47\%) were currently undergoing antitumor drug treatment, mostly at first line [10]. Overall use of maintenance therapy at first line was $12 \%$, which is higher than the $7 \%$ found in this study; but similarly, lenalidomide was the most commonly used first-line maintenance therapy $(45 \%)$ and second- and third-line agent overall [10]. Both studies suggest that first- and subsequent-line treatment practices are comparable across Europe; and that up-to-date recommendations are generally being followed [3,11]. Similar to the current study, Yong et al. 2016 (in a further analysis of the Raab et al. 2016 chart review) noticed that TTP decreased in later treatment lines [28]. The supportive treatments used by the patients were also comparable [28]. There is currently only limited published data on treatment patterns in newly diagnosed multiple myeloma patients; our study is providing important additions to current evidence.

In a literature search and systematic review of the economic evaluations of novel therapeutic agents in MM, Aguiar et al. 2016 argued that current economic evidence for novel agents is poor; only eight studies could be included and most found that novel agents were generally cost effective at a threshold of up to $\$ 100,000$ per quality-adjusted life year, but the reporting quality of the studies was low and there was large variation in methodologies [29]. This highlights the importance of the HCRU-associated cost analyses included in the current study.

The results reflect the lack of consensus over the standard of care or optimal choice of treatments for MM and how treatment practices are likely to evolve as more evidence is gathered on newer agents. Drug treatment choice depends on a number of factors including previous therapies, the individual characteristics of the patient and their disease status, and local guidelines and reimbursement policies. The approval of lenalidomide in this new indication is expected to increase the use of maintenance therapy post-ASCT. When considered in isolation, the increased adoption of lenalidomide maintenance therapy may appear to have cost implications; however, its use may offset overall costs in two main ways: it will delay relapse, saving hospital resources; and the choice of second-line treatment will be impacted, as patients who relapse on lenalidomide maintenance therapy will not proceed to lenalidomide-based triplet options in later lines of treatment.

Since 2015, new agents including the proteasome inhibitor ixazomib, monoclonal antibodies daratumumab and elotuzumab, and the histone deacetylase inhibitor panobinostat have been approved for the treatment of relapsed and/or refractory MM, and offer further treatment options that may change the therapeutic landscape again and help to avoid the need for multiple lines of therapy [30,31,32].

This study has a number of strengths. For example, it included multiple sites and countries, the setting was reflective of real-world practice and all MM patients were observed (with minimal screening criteria) rather than a subset of patients as would be the case in an interventional clinical trial. Understanding real-world practice is important when making decisions about new treatments, influencing future clinical studies and research and development; the data obtained from real-world practice provides insight into how new agents can be used in treatment pathways [10]. On the other hand, there are also a number of limitations that should be borne in mind when considering the results. The study included only a small sample of maintenance patients so the comparative results need to be interpreted cautiously. This may be a reflection of maintenance prescribing post-ASCT being a relatively recent treatment phenomenon; moreover, during the observation period, lenalidomide was not reimbursed as a maintenance treatment, having recently received EMA approval (February 2017) [3]. As with all studies of this type, a key limitation is the reliance on accurate and complete medical records and data collection. However, a number of quality control steps were incorporated into the design to minimize the impact of this. Also, pilot interviews with physicians were undertaken to ensure that the eCRFs were user friendly. The possibility of a physician selection bias is also a limitation; although steps were taken to mitigate this bias by recruiting a range of physicians from different regions, practice types and sizes, a prospective study is needed to address these issues. 


\section{Conclusion}

In conclusion, this study highlights the different treatment pathways that are employed post-ASCT and the extent of current maintenance therapy usage, which is likely to increase owing to the recent indication approval of lenalidomide for maintenance monotherapy treatment. The results suggest that patients who receive maintenance therapy at first line have delayed disease progression (as maintenance therapy prolongs the remission period compared with patients who do not receive any maintenance therapy). As the remission period is prolonged, future treatment lines will be deferred; previous research has shown that lenalidomide maintenance treatment achieves this without deterioration in health-related quality of life [33]. Furthermore, the prolonged remission may also spread the burden of HCRU along the treatment pathway (i.e., deferred costs of subsequent MM treatment and reducing the overall monthly HCRU cost relating to hospitalizations, HCP visits and monitoring), thus assisting healthcare services with the allocation of resources across patients. Further studies are needed to support these conclusions and to inform health economic analyses.

\section{Future perspective}

MM is a disease associated with vast clinical and genetic heterogeneity. Current research indicates miscellany in the management and treatment of MM, highlighting the need for alignment across clinical practice for patients.

As the life expectancy of our population increases, MM will undoubtedly become a disease primarily associated with the elderly. Long-term treatments are now increasingly being favored in practice; however, it is imperative to ensure optimum patient outcomes and efficient use of HCRU throughout the patient's life span. For example, the introduction of novel agents and the recently approved indication for lenalidomide, as a maintenance treatment, have proven to significantly increase both patient and clinical outcomes, specifically overall survival and PFS, for these patients when implemented within the early stages of treatment, in other words, first line.

However, this extension of life is associated with a further burden of increasing comorbidities, frailty and highrisk disease for this patient population; all of which can be under-represented in the results produced by large multicenter trials, necessitated by national funding bodies. Furthermore, studies have also shown that as patients relapse, the qualitative and quantitative burden increases. By treating patients earlier and keeping them in remission for longer during the early stages of treatment, the overall quality of the treatment experience is improved.

Further real-world data will be essential to assess how to most effectively utilize clinical interventions for these individuals in order for novel treatments to remain cost effective. Additional research could be conducted within the USA as maintenance therapy with lenalidomide post-transplant is currently the standard of care, to further quantify and validate the initial findings presented within this paper. The outlook for patients with MM has changed considerably over the last decade, and with the increasing adoption of novel agents, improving response rates and toxicity profiles, the field will continue to evolve.

\section{Summary points}

- The introduction of novel agents such as lenalidomide as maintenance therapy post autologous stem cell transplant (ASCT) represented a major advancement in the management of multiple myeloma.

- Study results found that a high proportion of the patient sample did not go on to receive further drug therapy post-ASCT. Of the patients who did receive maintenance therapy, majority were given lenalidomide monotherapy.

- Only a fifth of maintenance therapy patients progressed to second-line therapy, and none progressed to third line in the observation period.

- Patients receiving maintenance therapy took longer to progress from first line versus patients not receiving any maintenance treatment up until progression.

- Median time to progression was longer in patients who received maintenance therapy post-ASCT compared with those who did not.

- Data indicate that patients receiving lenalidomide maintenance therapy have a lower monthly healthcare resource utilization versus patients who do not receive any maintenance treatment.

\section{Acknowledgements}

The authors would like to thank N Milligan of Nancy Milligan Medical Ltd for preparing the draft manuscript on behalf of Adelphi Real World in accordance with the European Medical Writers Association guidelines. 
Financial \& competing interests disclosure

This study is supported by Celgene Corporation, Summit, NJ. J Ashcroft reports consultancy, honoraria and speaker's bureau for Celgene Corporation, Amgen, Janssen, Takeda; D Judge, G Taylor-Stokes and C Middleton report employment with Adelphi Real World; S Dhanasiri reports employment with and equity ownership. The authors have no other relevant affiliations or financial involvement with any organization or entity with a financial interest in or financial conflict with the subject matter or materials discussed in the manuscript apart from those disclosed.

Writing assistance was utilized in the production of this manuscript. Medical writing support was paid for by Adelphi Real World. The authors are fully responsible for all content and editorial decisions for this manuscript.

\section{Ethical conduct of research}

The authors state that they have obtained appropriate institutional review board approval or have followed the principles outlined in the Declaration of Helsinki for all human or animal experimental investigations.

\section{Open access}

This work is licensed under the Attribution-NonCommercial-NoDerivatives 4.0 Unported License. To view a copy of this license, visit http://creativecommons.org/licenses/by-nc-nd/4.0/

Supplementary data

To view the supplementary data that accompany this paper please visit the journal website at: www.futuremedicine.com/doi/full/10.2217/ijh-2018-0004

\section{References}

Papers of special note have been highlighted as: • of interest; $\bullet \bullet$ of considerable interest

1. Lehners N, Becker N, Benner A et al. Analysis of long-term survival in multiple myeloma after first-line autologous stem cell transplantation: impact of clinical risk factors and sustained response. Cancer Med. 7, 307-316 (2017).

2. Scott EC, Hari P, Sharma M et al. Post-transplant outcomes in high-risk compared with non-high risk multiple myeloma, a CIBMTR analysis. Biol. Blood Marrow Transplant. 22(10), 1893-1899 (2016).

3. Moreau P, San Miguel J, Sonneveld P et al. Multiple myeloma: ESMO Clinical Practice Guidelines for diagnosis, treatment and follow-up. Ann. Oncol. 28(Suppl. 4), iv52-iv61 (2017).

- Recent approval of lenalidomide indication in the maintenance treatment setting.

4. Palumbo A, Bringhen S, Ludwig H et al. Personalized therapy in multiple myeloma according to patient age and vulnerability: a report of the European Myeloma Network (EMN). Blood 118(17), 4519-4529 (2011).

5. Moreau P, Attal M, Facon T. Frontline therapy of multiple myeloma. Blood 125, 3076-3084 (2015).

6. Kumar S. Stem cell transplantation for multiple myeloma. Curr. Opin. Oncol. 21(2), 162-170 (2009).

7. Mohty M, Richardson PG, McCarthy PL, Attal M. Consolidation and maintenance therapy for multiple myeloma after autologous transplantation: where do we stand? Bone Marrow Transplant. 50(8), 1024-1029 (2015).

8. Torimoto Y, Shindo M, Ikuta K, Kohgo Y. Current therapeutic strategies for multiple myeloma. Int. J. Clin. Oncol. 20(3), 423-430 (2015).

9. Attal M, Lauwers-Cances V, Marit G et al. Lenalidomide maintenance after stem-cell transplantation for multiple myeloma. N. Engl. J. Med. 366(19), 1782-1791 (2012).

10. Raab MS, Cavo M, Delforge M et al. Multiple myeloma: practice patterns across Europe. Br. J. Haematol. 175(1), 66-76 (2016).

- A retrospective chart review conducted for multiple myeloma patients across Europe, using a similar methodology. The patient demographics and clinical characteristics in this study reflect the sample captured in Raab 2016, validating the representivity of our sample to European clinical practice.

11. Kumar SK, Callander NS, Alsina M et al. Multiple myeloma, version 3.2017, NCCN clinical practice guidelines in oncology. J. Natl Compr. Canc. Netw. 15(2), 230-269 (2017).

12. McCarthy PL, Holstein SA, Petrucci MT et al. Lenalidomide maintenance after autologous stem-cell transplantation in newly diagnosed multiple myeloma: a meta-analysis. J. Clin. Oncol. 35, 3279-3289 (2017).

-• Meta-analysis demonstrates and confirms the significant overall survival and progression-free survival benefit of using lenalidomide maintenance therapy post-autologous stem cell transplant in multiple myeloma patients when compared with no drug therapy or placebo.

13. McCarthy PL, Owzar K, Anderson KC et al. Phase III intergroup study of lenalidomide versus placebo maintenance therapy following single autologous stem cell transplant (ASCT) for multiple myeloma (MM): CALGB 100104. J. Clin. Oncol. 28(Suppl. 15), 8017 (2010). 
14. McCarthy PL, Owzar K, Hofmeister CC et al. Lenalidomide after stem-cell transplantation for multiple myeloma. N. Engl. J. Med. 366(19), 1770-1781 (2012).

15. Attal M, Cristini C, Marit G et al. Lenalidomide maintenance after transplantation for myeloma. J. Clin. Oncol. 28(Suppl. 15), 8018 (2010).

16. Koleva D, Cortelazzo S, Toldo C, Garattini L. Healthcare costs of multiple myeloma: an Italian study. Eur. J. Cancer Care (Engl.) 20(3), 330-336 (2011).

- Highlighting the economic burden of the treatment and management of multiple myeloma on the healthcare system.

17. Petrucci MT, Calabrese E, Levi A et al. Cost of illness in patients with multiple myeloma in Italy: the CoMiM study. Tumori 99(4), e193-e202 (2013).

18. Arikian SR, Milentijevic D, Binder G et al. Patterns of total cost and economic consequences of progression for patients with newly diagnosed multiple myeloma. Curr. Med. Res. Opin. 31(6), 1105-1115 (2015).

19. Pierelli L, Berto P, Accorsi $P$ et al. The costs of mobilisation and collection of peripheral blood stem cells in multiple myeloma and lymphoma in an European country: results from The Gruppo Italiano Trapianto Midollo Osseo (GITMO) and Società Italiana di Emaferesi e Manipolazione Cellulare (SIdEM) survey. Transfus. Apher. Sci. 49(3), 615-622 (2013).

20. Cook R. Economic and clinical impact of multiple myeloma to managed care. J. Manag. Care Pharm. 14(Suppl. 7), 19-25 (2008).

21. Meehan KR, Fitzmaurice T, Root L et al. The financial requirements and time commitments of caregivers for autologous stem cell transplant recipients. J. Support. Oncol. 4(4), 187-190 (2006).

22. Rifkin RM, Jagannath S, Durie BGM et al. E1277 analysis of the connect MM registry: treatment outcomes and healthcare resource utilization in patients with newly diagnosed multiple myeloma who received lenalidomide maintenance or no maintenance [Eposter presentation]. Presented at: 22nd Congress of the European Hematology Association. Madrid, Spain, 22-25 June 2017.

-• Analyzed registry data for patients who did and did not receive maintenance therapy. The data suggested that there is a reduction in healthcare resource utilization for patients receiving lenalidomide maintenance treatment versus patients who did not receive drug therapy post stem cell transplant.

25. StataCorp. Stata Statistical Software: Release 15. StataCorp LLC, College Station, TX, USA, 2017. www.stata.com/support/faqs/resources/citing-software-documentation-faqs/

26. European Medicines Agency. Revlimid assessment report EMA/CHMP/108277/2017. www.ema.europa.eu/docs/en_GB/document_library/EPAR_-_Assessment_Report_-_Variation/human/000717/WC500225259.pdf

27. Tolerability and efficacy of post transplant lenalidomide maintenance therapy in multiple myeloma: a real world single centre experience. Blood J. www.bloodjournal.org/content/130/Suppl_1/3462?sso-checked=true

28. Yong K, Delforge M, Driessen C et al. Multiple myeloma: patient outcomes in real-world practice. Br. J. Haematol. 175(2), 252-264 (2016).

29. Aguiar PM, Lima TM, Storpirtis S. Systematic review of the economic evaluations of novel therapeutic agents in multiple myeloma: what is the reporting quality? J. Clin. Pharm. Ther. 41(2), 189-197 (2016).

30. Leblebjian H. Navigating new treatment paradigms in relapsed or refractory multiple myeloma. Presented at: 13th Hematology/Oncology Pharmacy Association Annual Conference. Anaheim, CA, USA, 29 March-1 April 2017.

31. Larocca A, Mina R, Gay F, Bringhen S, Boccadoro M. Emerging drugs and combinations to treat multiple myeloma. Oncotarget (2017) (Epub ahead of print). doi: 10.18632/oncotarget.19269.

32. Sonneveld P, De Wit E, Moreau P. How have evolutions in strategies for the treatment of relapsed/refractory multiple myeloma translated into improved outcomes for patients? Crit. Rev. Oncol. Hematol. 112, 153-170 (2017).

33. Abonour R, Durie BGM, Jagannath S et al. 537 health-related quality of life of patients with newly diagnosed multiple myeloma receiving any or lenalidomide maintenance after autologous stem cell transplant in the Connect MM Disease Registry [oral abstract]. Presented at: ASH 58th Annual Meeting \& Exposition. San Diego, CA, USA, 3-6 December 2016. 